\title{
PRINCÍPIOS ATIVOS UTILIZADOS NO TRATAMENTO DO MELASMA ATRAVÉS DO MICROAGULHAMENTO
}

\author{
Danyla Alves dos Santos ${ }^{1}$ \\ Ana Cristina Silva de Santana ${ }^{2}$ \\ Alana Santiago Costa de Santana ${ }^{3}$ \\ Paula Souza Santana Santos ${ }^{4}$ \\ Louise Rodrigues Barreto ${ }^{5}$
}

RESUMO: O presente estudo trata de uma revisão bibliográfica sistemática associada aos princípios ativos utilizados no tratamento do melasma através da técnica do microagulhamento. Foi realizado um levantamento bibliográfico por meio do pubmed com o objetivo de alcançar uma melhor compreensão do tema e verificar o efeito dos ativos utilizados na indução percutânea, a revisão evidencia que diferente tratamento tem sido utilizado através dos ácidos retinóico e tranexânico, e a hidroquinona para vários tipos de macolas na pele, mostrando eficácia e satisfação no resultado.

Palavras-chave: Melasma. Microagulhamento facial.

ABSTRACT: This study is a systematic literature review associated with the active principles used in the treatment of melasma through the microneedling technique. in percutaneous induction, the review shows that different treatments have been used through retinoic and tranexanic acids, and hydroquinone for various types of skin moles, showing efficacy and satisfaction in the result.

Keywords: Melasma. Microneedlin facial.

\section{INTRODUÇÃO}

De acordo Lima, Lima e Takano (2013), os iniciantes a utilizar o procedimento de microagulhamento foram Orentreich e Orentreith com a aplicação de agulhas com

\footnotetext{
I Graduação em Farmácia, Unifacs/ Feira de Santana-BA. danylasantos24@yahoo.com

${ }^{2}$ Graduação em Farmácia, Unifacs/Feira de Santana-BA. E-mail- anacris305@hotmail.com

${ }^{3}$ Graduação em Farmácia, Unifacs/ Feira de Santana- BA. E- mail- Alanasantiago@hotmail.com

${ }^{4}$ Graduação em Farmácia, Unifacs/Feira de Santana-BA. E-mail paula.lay25@gmail.com

5 Orientadora. Graduação em Biomedicina UESC/ Ilheus-BA. E-mail-louise.barreto@unifacs.br
} 
o propósito de ativar a geração de colágeno na terapêutica de cicatrizes deprimidas e rugas, técnica difundida com o nome de subincisão.

O microagulhamento incita a criação de colágeno na pele diante de uma influência automática, com a utilização de um rolo composto por microagulhas. Esse rolo é constituído de polietileno e formado por agulhas de aço inoxidável e estéreis, as quais são dispostas proporcionalmente em fileiras, numa quantidade que varia entre I92 e 540 unidades, a depender do resultado e finalidade do tratamento. O comprimento das microagulhas podem variar de $0,25 \mathrm{~mm}$ a 2,5 $\mathrm{mm}$ de diâmetro (PIATTI, 2013).

O maior órgão do corpo humano é a pele, responsável pela relação entre o meio interior e exterior conferindo a proteção e colaborando para o bom funcionamento do organismo (KALAFA, 2017). Possui três camadas denominadas epiderme, derme e hipoderme, chamada também de tecido subcutâneo (TORTORA, 2016). Na camada basal (hipoderme) a qual é a camada mais profunda, encontram-se os melanócitos, células responsáveis pela nossa pigmentação, sua principal função é a produção de melanina (SILVA, 2016). Os melanócitos encontram-se reservados nos melanossomas dentro dos queratinócitos. O excesso da melanina causa uma doença conhecida como melasma, (NASCIMENTO, 2019).

O melasma é uma hiperpigmentação cutânea, ou seja, manchas com tonalidades de várias cores ou até mesmo pontos escuros, que se caracterizam por manchas com as mesmas formas, medidas e mesma posições umas em conformidades com as outras, e que pode atingir tantas pessoas do sexo feminino como masculino, sendo mais encontrada nas mulheres em idade fértil. Geralmente o melasma pode ser observado principalmente na face (MASCENA, 2016).

Os princípios ativos como ácido retinoico aumenta a síntese do colágeno diminuindo a produção da colagenose, tranexanico que atua na melanogênese por ser inibidor da plasmina e a hidroquinona inibindo a tirogenase convertendo dopa em melanina, são agentes que apresenta mecanismo de ação no clareamento do melasma, associado ao microagulhamento. 
O presente trabalho tem como objetivo geral demonstrar os princípios ativos com maior eficácia no tratamento do melasma através do microagulhamento, na atualidade. Diante do tema abordado os objetivos específicos são: Abordar os ácidos mais utilizados no tratamento do melasma através do microagulhamento; comparar a eficácia do microagulhamento com novas técnicas utilizada no tratamento do melasma.

\section{METODOLOGIA}

Este estudo trata-se de uma revisão bibliográfica sistemática. De acordo com Cook et al. (1997), a revisão bibliográfica sistemática é identificada por ser regular, exata e aplicável.

Essa pesquisa teve como base de dados a PubMed, em que a busca foi feita utilizando as palavras chaves melasma, microagulhamento facial, facial microneedlin, nos idiomas português e inglês. Os levantamentos dos estudos referentes ao tema escolhido priorizaram o ano de 2021, para que a pesquisa contasse com dados mais recentes. Além disso, foram pesquisados livros técnicos específicos ao tema.

Os critérios de inclusão foram os artigos do ano de 202I, que continham as palavras chaves supracitadas, artigos de meta-análise, com ensaio clinico experimentais que obtinham resultados no tratamento das substâncias associada ao microagulhamento, como ácido tranexâmico, retinoico, hidroquinona, plasma.

Os critérios de exclusão foram artigos publicados antes do ano de 2021, artigos que abordavam o tratamento do melasma em período gestacional por causar malformação fetal, e estudos que relatavam sobre o tratamento utilizando apenas o microagulhamento.

\section{RESULTADO E DISCUSSÃO}

Ao realizar pesquisa na plataforma cientifica supramencionada, analisando os critérios de elegibilidade dos artigos encontrados, após análise do título e resumo foram requisitados 92 artigos que correspondem de forma especifica ao tema abordado: 
tratamento do melasma através do Microagulhamento. Segue abaixo tabela da seleção dos artigos selecionados. Figura I: FLUXOGRAMA PARA SELEÇÃO DOS ARTIGOS

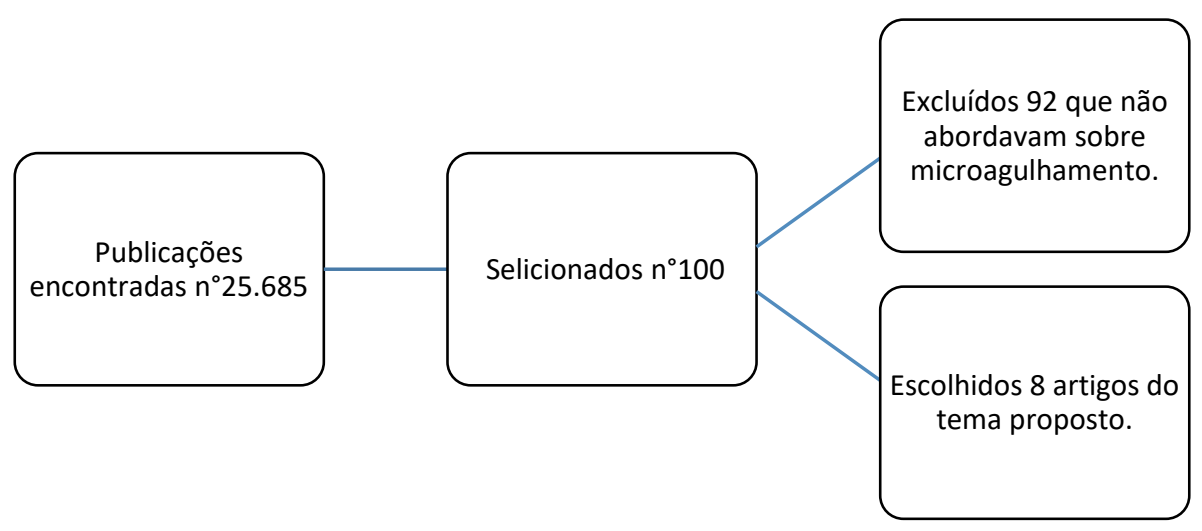

Figura 2: LISTA DOS ARTIGOS SELECIONADOS.

\begin{tabular}{|l|l|l|l|}
\hline Título do estudo & Autor/Ano & Publicação & Objetivo \\
\hline $\begin{array}{l}\text { Estudo randomizado, duplo-cego, } \\
\text { controlado por placebo, de dupla face, da } \\
\text { eficácia do ácido tranexâmico por } \\
\text { administração de medicamento por } \\
\text { microagulhamento no tratamento de } \\
\text { melasma; }\end{array}$ & $\begin{array}{l}\text { Dâmia et al } \\
\text { (202I); }\end{array}$ & $\begin{array}{l}\text { JCD. J Cosmet } \\
\text { Dermatol }\end{array}$ & $\begin{array}{l}\text { Avaliar a eficácia do ácido } \\
\text { tranexâmico quando aplicado } \\
\text { na forma de liberação de } \\
\text { fármacos por } \\
\text { microagulhamento no } \\
\text { tratamento do melasma facial; }\end{array}$ \\
\hline $\begin{array}{l}\text { Solução de ácido tranexâmico tópico } \\
\text { assistida por microagulhamento versus } \\
\text { hidroquinona a 4\% para o tratamento de } \\
\text { melasma: um estudo randomizado de } \\
\text { dupla face; }\end{array}$ & $\begin{array}{l}\text { Mohamed et } \\
\text { al(202I); }\end{array}$ & $\begin{array}{l}\text { JCD. J Cosmet } \\
\text { Dermatol }\end{array}$ & $\begin{array}{l}\text { Neste estudo, objetivamos } \\
\text { avaliar e comparar a eficácia do } \\
\text { ácido tranexâmico aplicado } \\
\text { topicamente após o } \\
\text { microagulhamento versus } \\
\text { hidroquinona (HQ) } \\
\text { aplicada topicamente sozinha } \\
\text { em pacientes com melasma; }\end{array}$ \\
\hline $\begin{array}{l}\text { Micro-agulhamento versus laser de } \\
\text { dióxido de carbono fracionado para } \\
\text { administração de ácido tranexâmico no } \\
\text { tratamento do melasma: um estudo de } \\
\text { face dividida; }\end{array}$ & $\begin{array}{l}\text { Khaled MM } \\
\text { al (202I); }\end{array}$ & $\begin{array}{l}\text { JCDawy et } \\
\text { Dermatol Cosmet }\end{array}$ & $\begin{array}{l}\text { O objetivo deste estudo foi } \\
\text { comparar a utilização do laser } \\
\text { de microagulhas e de CO } 2 \\
\text { fracionado para a liberação de } \\
\text { fármacos de TXA no } \\
\text { tratamento do melasma facial; }\end{array}$ \\
\hline
\end{tabular}




\begin{tabular}{|c|c|c|c|}
\hline $\begin{array}{l}\text { Eficácia e segurança do ácido } \\
\text { tranexâmico no tratamento do melasma } \\
\text { adulto: uma meta-análise atualizada de } \\
\text { ensaios clínicos randomizados; }\end{array}$ & $\begin{array}{l}\text { Xiaowei } \\
\text { Feng MD et } \\
\text { al (2021) }\end{array}$ & $\begin{array}{l}\text { Journal of } \\
\text { Clinical } \\
\text { Pharmacy and } \\
\text { Therapeutics }\end{array}$ & $\begin{array}{l}\text { O Melasma afeta } \\
\text { significativamente a aparência e } \\
\text { a qualidade de vida dos } \\
\text { pacientes. Embora várias } \\
\text { modalidades terapêuticas } \\
\text { tenham sido tentadas para } \\
\text { tratar o melisma, incluindo } \\
\text { ablação a laser, peeling químico } \\
\text { e agentes despigmentantes } \\
\text { usados para clarear o melasma } \\
\text { em algum grau, nenhum } \\
\text { procedimento ou agente } \\
\text { universalmente eficaz garante } \\
\text { resultados satisfatórios. No } \\
\text { entanto, médicos e pacientes } \\
\text { estão especialmente } \\
\text { preocupados com a } \\
\text { hiperpigmentação pós- } \\
\text { inflamatória causada pelo laser; }\end{array}$ \\
\hline $\begin{array}{l}\text { Uso do ácido retinóico associado ao } \\
\text { microagulhamento no tratamento do } \\
\text { melasma epidérmico: eficácia e } \\
\text { parâmetros de estresse oxidativo; }\end{array}$ & $\begin{array}{l}\text { Clarissa LM } \\
\text { da Silva } \\
\text { Bergmann } \\
\text { et al (2021) }\end{array}$ & $\begin{array}{l}\text { Arch } \\
\text { Dermatol Res }\end{array}$ & $\begin{array}{l}\text { Avaliar a eficácia do tratamento } \\
\text { isolado com ácido retinóico e } \\
\text { sua combinação com a técnica } \\
\text { de microagulhamento no } \\
\text { melasma facial, buscando } \\
\text { associar esses resultados a } \\
\text { possíveis danos oxidativos; }\end{array}$ \\
\hline $\begin{array}{l}\text { A eficácia das diluições orais versus } \\
\text { diferentes diluições de microinjeções de } \\
\text { ácido tranexâmico intradérmico em } \\
\text { ensaio clínico randomizado de melasma- } \\
\text { A }\end{array}$ & $\begin{array}{l}\text { Heba El } \\
\text { Hadidi et al } \\
(\text { 202I) }\end{array}$ & Dermatol Ther & $\begin{array}{l}\text { Avaliar e comparar a eficácia de } \\
\text { diluições orais versus diferentes } \\
\text { diluições de TXA intradérmico } \\
\text { no melasma. Um total de } 45 \\
\text { pacientes do sexo feminino com } \\
\text { melasma foram aleatoriamente } \\
\text { e igualmente designados a três } \\
\text { grupos de tratamento. }\end{array}$ \\
\hline $\begin{array}{l}\text { Eficácia e segurança do plasma rico em } \\
\text { plaquetas no melasma: uma revisão } \\
\text { sistemática e meta-análise }\end{array}$ & $\begin{array}{l}\text { Lingyun } \\
\text { Zhao et al } \\
(2021)\end{array}$ & $\begin{array}{l}\text { Dermatologia } \\
\text { e Terapia }\end{array}$ & $\begin{array}{l}\text { objetivo de estudar a eficácia e } \\
\text { segurança do PRP no } \\
\text { tratamento de pacientes com } \\
\text { melasma quando usado sozinho } \\
\text { ou como terapia adjuvante e, } \\
\text { esperançosamente, fornecer } \\
\text { novas evidências clínicas para o } \\
\text { tratamento do melasma } \\
\end{array}$ \\
\hline $\begin{array}{l}\text { Efeito sinérgico de } 300 \mu \mathrm{m} \text { de } \\
\text { radiofrequência fracionada de } \\
\text { microagulação de profundidade de agulha } \\
\text { no tratamento da hiperpigmentação } \\
\text { cutânea induzida por envelhecimento } \\
\text { induzida por senescência. }\end{array}$ & $\begin{array}{l}\text { Young em } \\
\text { Lee et al. } \\
(202 \mathrm{I}) \text {. }\end{array}$ & $\begin{array}{l}\text { International } \\
\text { Journal of } \\
\text { Molecular } \\
\text { Sciences }\end{array}$ & $\begin{array}{l}\text { O efeito sinérgico do novo } \\
\text { tratamento de radiofrequência } \\
\text { fracionada com microagulação } \\
\text { (FMR) de } 300 \mu \mathrm{m} \text { de } \\
\text { profundidade de agulha e laser } \\
\text { Q-switched ND: YAG } \\
\text { convencional na } \\
\text { hiperpigmentação da pele } \\
\text { associada ao envelhecimento }\end{array}$ \\
\hline
\end{tabular}


O Microagulhamento também é nomeado de terapia de indução percutânea de colágeno (TIPC), essa técnica é aplicada por um equipamento chamado derma roller, existe também o dispositivo eletrônico. O equipamento composto por um rolo de polietileno encravado por agulhas, que tem entre 190 e 450 agulhas (NEGRÃO, 2016).

O microagulhamento ou indução percutânea de colágeno (IPC) apresenta resultado eficaz no tratamento do melasma, tem benefício devido a ser feito de maneira repentina, pequeno custo e acessível manejo no local com dificuldade em manejar. Ora o microagulhamento está sendo usado com principais ativos farmacológicos para obtenção de expandir e transpassar a derme, com a inserção de vários ativos, com a finalidade de obter o efeito do processo terapêutico. (PALHETA, 2017).

Os Ativos mais utilizados nessa revisão de literatura sistemática indicaram o ácido retinóico ou tretanoína, conhecida como vitamina $\mathrm{A}$ ácida, lipossolúvel e para ser transformada necessita da proteína (CRABP), através do efeito esfoliativo e dispensando os grânulos dentro dos queratinocitos que facilita a eliminação através do aumento do tunover epidérmico. (DREPREZ, 2009).

A esfoliação aumentada no extrato córneo facilita a drenagem dos pilossebáceas, reduz o tempo de exposição entre os queratinocitos e malanocitos, modulando as funções do colágeno tipo i nas células quiescentes "células sem meiose", já o colágeno de tipo IV é estimulado quando utilizado esse ativo, promovendo a perda rápida do pigmento, aumenta a fagocitose da melanina pelos macrófagos. $\mathrm{O}$ mecanismo de ação dos ácidos retinóico é interromper e/ou estimular as reações nós queratinocitos, regulando através da ligação a receptores nucleares, transformando o retinol em ácido retinóico (ALAM et al, 2010).

A hodroquinona é um derivado fenólico, com estrutura similar com o precursor da melanina, na presença de dopa compete com a tirosina que é o substrato da tirosinase inibindo a síntese da melanina, atua na degradação dos melanossomos com efeito clareador. (MONTEIRO, 2012). 
O ácido tranexâmico sugeriu uma redução da atividade tirosinase que na presença de queratinócitos consegue desempenhar a síntese de melanina por inibir os ativadores dos melanocitos contidos na cultura de queratinocitos condicionados. A principal função é bloquear a conversão do plasminogenio em plasmina ativa a secreção de precursores da fosfolipase $\mathrm{A}_{2}$ que produz o ácido aracdonico e induz a liberação do crescimento dos fibroblastos.

Para Dâmia Kuster et al (2021), em seu estudo prioriza a avaliação do uso de ácido tranexâmico através da micropunturas utilizadas para indução percutânea de colágeno provocada na pele, com tamanho adequado das agulhas através do derma holer para atingir a camada mais externa (derme) da pele. Já para Mohamed et al (2021), evidenciou em sua pesquisa, que o uso tópico do mesmo fármaco após a terapia de indução de colágeno através do microagulhamento apresentou eficácia na redução das manchas de melasma.

Khaled MM Mekawy et al (2021), publicou artigo sobre o dióxido de carbono $\left(\mathrm{CO}_{2}\right)$ fracionado à laser e microagulhamento. $\mathrm{O}$ ácido tranexâmico é usado nas vias oral, tópica e intradérmica, o estudo realizado com 30 mulheres, com melasma facial bilateral, submetidas a microagulhas de um dos lados da face e do outro lado, foi utilizado laser de $\mathrm{CO}_{2}$ fracionado, após o procedimento, foi administrado ácido tranexâmico $4 \mathrm{~g} / \mathrm{ml}$ em toda a face, esse método contou com um total de 6 sessões administrada quinzenalmente em cada paciente. Em ambos tratamentos mostraram eficácia da hiperpigmentação e segurança na administração do ácido.

No trabalho apresentado por Xiaowei Freng MD et al (202I), a utilização do ácido tranexâmico puro, como adjuvante no tratamento contra o melasma. $\mathrm{O}$ ácido tranexâmico tem efeito clareador e anti-inflamatório, ao ser injetado por via intradérmica através do microagulhamento, a plasmina que é via de bloqueio do sítio de ligação da lisina no sítio da célula, faz com que reduza a produção de prostaglandina, e consequentemente redução de melanogênese nos melanócitos, com resultados fidedignos a melhora do melasma. 
Conforme Clarissa (202I), elaborou uma pesquisa com 42 mulheres por 2 meses, sendo que a cada is dias eram realizados os procedimentos conforme cada estabelecido para cada grupo. A divisão A e B onde, o grupo A realizou microagulhamento e ácido retinóico e o Grupo $\mathrm{B}$, utilizou apenas ácido retinóico 5\% tópico. Houve redução de melasma em ambos os grupos, porém foi verificado aumento do extress oxidativo celular através do (Carbonil) nos 30 dias. $\mathrm{O}$ autor ressalta que o microagulhamento não foi tão eficaz com o princípio ativo utilizado por reduzir a defesa do antioxidante.

De acordo com Heba El Hadide et al (2021) utilizou ácido tranexâmico com diferentes vias de administração (oral e intradérmica), o estudo contou com 45 mulheres com melasma com acompanhamento total de 8 semanas, esse montante foi dividido em 3 grupos de 15 pessoas, cada grupo foi nomeados, em grupo A que utilizou o princípio ativo de forma oral 25omg. O grupo B utilizou intradérmico $100 \mathrm{mg} / \mathrm{ml}$. O grupo $\mathrm{C}, 4 \mathrm{mg} / \mathrm{ml}$, a pesquisa demonstrou que todos os três grupos tiveram êxito por reduzir o melasma, sendo mais significativo a intradérmica independente da dosagem.

$\mathrm{O}$ artigo foi utilizado Plasma rico em plaquetas (PRP) em 395 pacientes com melasma, foram divididos três grupos de acordo com o índice de área e gravidade do melasma (MASI), com tratamento de duração entre 4 a 12 semanas. Foram utilizados três regimes terapêuticos para tratamento do PRP, micro injeção, microagulhamento e injeção intradérmica. Dentre as terapias informadas, o microagulhamento e a injeção intradérmica obteve maior índice de satisfação.

O ensaio clínico de Young em Lee et al (2021), com 25 mulheres, onde houve divisão de dois grupos, um grupo realizou análise de radiofrequência de microagulhamento fracionado (FMR) com a profundidade recomendado de $300 \mu \mathrm{m}$ e outro grupo usou laser Q-switched ND:YAG (QSNY) e FMR como adjuvante do tratamento. O microagulhamento tem eficácia no tratamento do melasma, já o microagulhamento como adjuvante do laser teve uma atividade mais intensa e melhora significativa na destruição do melanócito, com o objetivo de explorar o 
mecanismo fisiológico no tratamento, que demonstrou resultados positivos na hiperpigmentação, com redução do índice de melanina.

\section{CONCLUSÃO}

Devido ao fato do seu aparecimento na face, o melasma causa uma série de insatisfações e problemas na qual ao indivíduo acometido pela doença, vai se sentir inferiorizado diante do seu meio de convívio causando estresse psicológicos e assim comprometendo sua qualidade de vida. Em virtude do seu descontentamento com a aparência, vão surgindo inquietações relacionadas com a autoestima dos pacientes, refletindo na vida pessoal e profissional (HANDEL, 2013).

Essa revisão bibliográfica sistemática correlacionou publicações de diferentes autores, associada aos princípios ativos utilizados no tratamento do melasma através da técnica do microagulhamento. A revisão evidenciou diferentes tratamentos utilizados através dos ácidos retinóico e tranexâmico, e a hidroquinona para vários tipos de hiperpigmentação na pele, mostrando eficácia e satisfação no resultado de todos os casos abordados nessa revisão.

\section{ऽ REFERÊNCIAS}

ALAM, Murad; GLADSTONE, Hayes B.; TUNG, Rebeca C. Dermatologia Cosmética. Rio de Janeiro,2oro;

DÂMIA, Kuster Kaminski Arida I, Priscila Regina Orso Rebellato I, Giovana Liz Marioto de Campos I, Adriane Costa I, Juliano Vilaverde Schmitt 2, Thelma Larocca Skare 3, Luciana Rodrigues Lisboa Faucz I. doi: Io.III / jocd.I4257. PMID: 34077619 DOI: Io.IIII / jocd.I4257; J Cosmet Dermatol,.2 de junho de 202I.

DEPREZ, Philippe M. D. Peeling químico superficial, médio e profundo. Rio de Janeiro: Revinter LTda, 2009; 
CLARISSA, LM da Silva Bergmann et al. Uso do ácido retinóico associado ao microagulhamento no tratamento do melasma epidérmico: eficácia e parâmetros de estresse oxidativo, Arch Dermatol Res. 202I.

COOK, D.J.; Mulrow, C.D.; Haynes, R.B. Systematic reviews: synthesis of best evidence for clinical decisions. Annals of Internal Medicine, v.126, n.5, pp.376-38o, 1997;

HANDEL,Ana Carolina; MIOT,LucianeDonidaBartoli; MIOT, HelioAmante.Melasma: a clinicalandepidemiologicalreview (nih.gov), Anais brasileiros de dermatologia, v, 89, 5, p.771 - 772, 2014.

HEBA, El Hadidi, Rana Mosaad, Nanis Ragab ,The efficacy of oral vs different dilutions of intradermal tranexamic acid microinjections in melasma-A randomized clinical trial, doi: ro.III/dth.14924, 2021. Diponivél em: https://pubmed.ncbi.nlm.nih.gov/33651442. Acessado em setembro de 2021.

KALAFA, EA Growney et al. 9.I. I Skin anatomy and physiology. Electrospun Materials for Tissue Engineering and Biomedical Applications: Research, Design and Commercialization, p. 179, 2017;

KHALED, MM Mekawy et al. ;20(2):460-465. doi: Io.IIII/jocd.13537. Microagulhamento versus laser de dióxido de carbono fracionado para administração de ácido tranexâmico no tratamento do melasma: um estudo de face dividida, J Cosmet Dermatol . 2021:

LIMA, E. V. A.; LIMA, M. A. TAKANO, D. Microagulhamento: estudo experimental e classificação da injúria provocada. SurgCosmet Dermatol.5(2):1ro-4. 2013;

LINGYUN, ZHAO ,Meng Hu ,Qing Xiao ,Runke Zhou ,Yu Li ,Lidan Xiong \& Li Li; Efficacy and Safety of Platelet-Rich Plasma in Melasma: A Systematic Review and Meta-Analysis, Dermatology and Therapy volume II, pagesi587-I597 (2021);

MOHAMED, S Zaky, Zakaria M Obaid, Eman A Khalil, Mohamed L Elsaie, Microneedling-assisted topical tranexamic acid solution versus $4 \%$ hydroquinone for 
treating melasma: A split-face randomized study; PMID: 34525492 -

DOI: ro.IIII/jocd.I4440 J Cosmet Dermatol, 202I;

MONTEIRO, Érica O. Melasma: abordagem tópica. R.B.M. Especial Cosmiatria 2. Moreira J.R. Editora, V.69, jun. 2012;

MASCENA, Thereza Cristyna Feitosa. MELASMAS E SUAS PRINCIPAIS FORMAS DE TRATAMENTO. 2016. $47 \mathrm{f}$. Monografia (Especialização) - Curso de Biomedicina Estética, Instituto Nacional de Ensino Superior e Pesquisa e Centro de Capitação Educacional, Recife, 2016;

NASCIMENTO, Débora Barbosa et al. Etiologia e tratamento medicamentoso de melasmas durante a gestação. Revista de Iniciação Científica e Extensão, v. 2, n. 3, p. 176-180, 2019;

NEGRÃO,o MMC. Microagulhamento, bases fisiológicas e práticas. São Paulo: CR8; 2016;

PALHETA, C. S. A.; et. al. Efeito do óleo de copaíba associado ao microagulhamento na pele de ratos: um estudo comparativo. Surgical e CosmeticDermatology, v.9, 2017;

PIATTI, I. L. Microagulhamento e fatores de crescimento. Revista Personalité, São Paulo, ano I6, n. 8, p. 22-25, 2013;

SILVA, Carolina Rufino de Sá. Estudo da utilização do ácido hialurónico como veículo de entrega de células autólogas em substitutos de pele. Tese de Doutorado, 2016;

TORTORA, Gerard J.; DERRICKSON, Bryan. Corpo Humano-: Fundamentos de Anatomia e Fisiologia. Artmed Editora, 2016;

Xiaowei Feng MD, et al. J Clin Pharm Ther; Eficácia e segurança do ácido tranexâmico no tratamento do melasma adulto: uma meta-análise atualizada de ensaios clínicos randomizados. 2021.Disponível em https://doi.org/ro.III/jcpt.13430, Acessado em 04 de outubro de 2021.

Young em Lee et al. Int J Mol Sci nt. J. Mol. Sci. 2021 , 22 (14), 7480; Disponivél em: Acessado em setembro2021, https://doi.org/I0.339o/ijms22147480 